\title{
Estrategias en el manejo individualizado de la hemorragia perioperatoria
}

Pérez Herrero MA, Sánchez Miguel A.

Hospital Clínico Universitario de Valladolid.

\section{Resumen}

Los anestesiólogos debemos participar en programas multidisciplinares y acordes a las circunstancias locales, de ahorro de sangre, cuyo objetivo es adoptar la actitud transfusional más eficiente y segura, optimizando el tratamiento del paciente quirúrgico. Nos propusimos como objetivo revisar las recomendaciones actuales en ahorro de sangre.

\section{Introducción}

Los anestesiólogos debemos participar en programas multidisciplinares $\mathrm{y}$ acordes a las circunstancias locales de ahorro de sangre, cuyo objetivo es adoptar la actitud transfusional más eficiente y segura, optimizando el tratamiento del paciente quirúrgico. Nos propusimos como objetivo realizar una revisión crítica y formativa de las recomendaciones actuales en ahorro de sangre en entorno perioperatorio.

\section{Metodología}

Se hizo una búsqueda en noviembre de 2017 en la base de datos Medline (Pubmed) utilizando como criterio de búsqueda "patient blood management" and "surgery" and "implementation". Se eligieron los trabajos más actualizados (en los últimos 5 años), revisiones sistemáticas o guías clínicas o documentos de consenso.

\section{Resultados}

Encontramos 743 trabajos de artículos y guías clínicas, de los que seleccionamos
54 resúmenes que comparaban diversas medidas ahorradoras de sangre, y 14 artículos para revisar a texto completo, que realizaban revisiones y guías clínicas de actuación.

Se han descrito más de 100 medidas en los programas individualizados de manejo de sangre $\mathrm{y}$ hemoderivados (Patient Blood Management). Todos los protocolos presentan características comunes: 1) multidisciplinares, 2) criterios transfusionales restrictivos, 3) evitan la transfusión, 4) protocolos de transfusión en caso de hemorragia masiva y 5) disponibilidad de productos sanguíneos. Casi todos los estudios revisados centran las recomendaciones en tres pilares fundamentales: la optimización de la masa eritrocitaria, minimizar las pérdidas sanguíneas y aumentar la tolerancia fisiológica a la anemia. Estas medidas se realizan tanto en el periodo preoperatorio, como en el intraoperatorio y postoperatorio, tal como se resumen en la tabla I.

Los programas de ahorro se basan en tres pilares: optimizar masa eritrocitaria, minimizar pérdidas sanguineas y mejorar la tolerancia fisiológica a la anemia. 


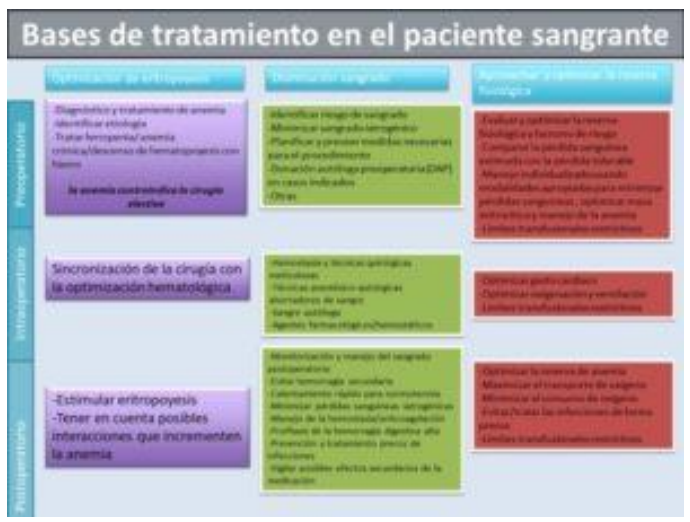

Tabla I. Pilares del tratamiento del paciente quirúrgico con riesgo de sangrado (modificado de Eelex A, Baikady RR. Perioperative Blood Management. Indian J Anaesth 2017; 61 (6): 456-462).

\section{ERRORES FRECUENTES A} EVITAR:

1. Utilizar transfusiones para tratar hipovolemia.

2. Umbral de transfusión liberal ( $\mathrm{Hb} 9 \mathrm{o}$ $10 \mathrm{mg} / \mathrm{dl})$.

3. Tratar anemia postoperatoria con hierro oral (menos eficaz que el iv).

\section{Detección y tratamiento de anemia preoperatoria}

La anemia preoperatoria es un factor de riesgo independiente de alargamiento de la estancia hospitalaria, complicaciones, necesidad de transfusión y aumento de mortalidad. En la visita preoperatoria se evalúa el estado clínico del paciente y se prevé el riesgo transfusional (el principal factor predictivo de transfusión es la hemoglobina) y se elige la técnica de ahorro de sangre y de optimización de hemoglobina en caso necesario. La OMS define anemia como una concentración de $\mathrm{Hb}$ menor de 13 $\mathrm{gr} / \mathrm{dl}$ en hombres, de $12 \mathrm{gr} / \mathrm{dl}$ en mujeres y de $11 \mathrm{gr} / \mathrm{dl}$ en embarazadas.

\subsection{Hierro intravenoso (iv)}

Indicado en pacientes con anemia ferropénica.

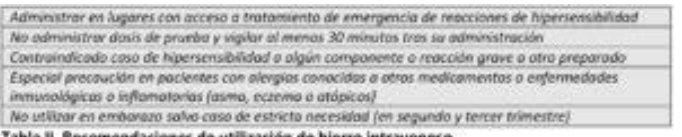

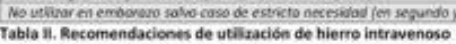

1.2. Eritropoyetina humana
recombinante (rHuEPO)

La EPO (eritropoyetina) es un factor de crecimiento eritropoyético que se produce en respuesta a la hipoxia o baja disponibilidad tisular de oxígeno. La rHuEPO se utiliza a dosis mínima efectiva (unos $40.000 \mathrm{U}$ semanales) y de forma individualizada en función de hemoglobina $(\mathrm{Hb})$ preoperatoria, peso del paciente, y $\mathrm{Hb}$ objetivo ajustada a pérdidas previstas. No hay que olvidar asociarla a hierro intravenoso, monitorizar respuesta y posibles complicaciones vasculares $\mathrm{o}$ hemodinámicas; y garantizar profilaxis antitrombótica adecuada, con las indicaciones siguientes:

a. Aumentar hemoglobina preoperatoria (cirugía ortopédica programada).

b. Facilitar extracciones para donación de sangre autóloga preoperatoria (más de 3 unidades).

c. Reducir transfusión alogénica.

\subsection{Donación de sangre autóloga preoperatoria $(D A P)$}

Únicamente indicada en cirugías programadas (con más de 3 semanas de antelación) que necesiten transfusión mayor de $30-40 \%$ o reserva de más de 3 unidades de sangre.

\section{Minimizar pérdidas sanguíneas} Protocolos actualizados de reversión de antiagregación-anticoagulación, plan anestésico y medidas terapéuticas individualizadas en función de las características del paciente y del procedimiento quirúrgico. Se aconseja la mínima dosis eficaz de antiagregación (AAS 100 mg/24 horas) 
o terapias puente en pacientes anticoagulados, y reintroducción precoz de anticoagulación/antiagregación en postoperatorio, una vez controlada la hemostasia.

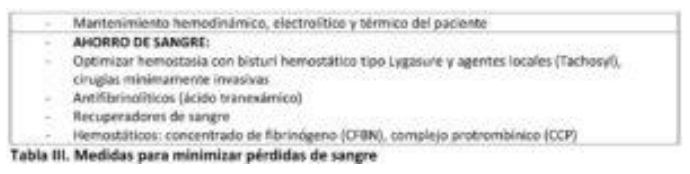

El manejo de la hemorragia quirúrgica en pacientes anticoagulados dependerá de su gravedad, tal como se esquematiza en las tablas IV y V.

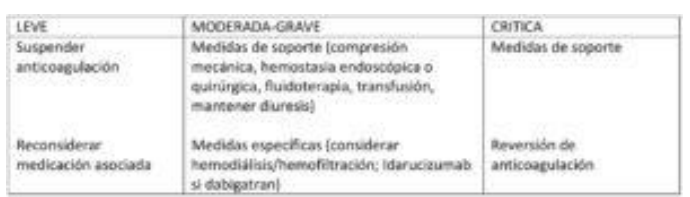

Tabla IV. Manejo de la hemorragia quirúrgica en función de su gravedad.

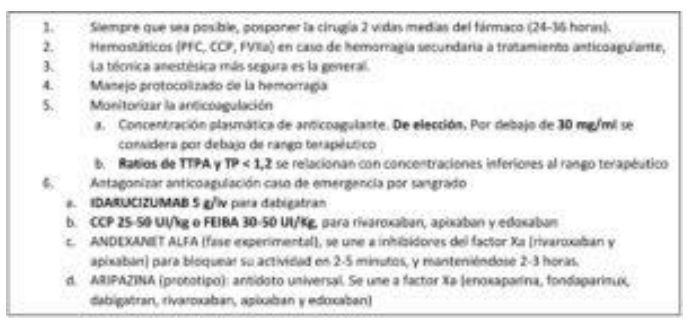

Tabla V. Medidas generales en el paciente quirúrgico anticoagulado.

\subsection{Fármacos hemostáticos}

\subsubsection{Antifibrinolíticos (Ácido tranexámico (ATX)).}

Utilidad demostrada en cirugía ortopédica (artroplastia de cadera, rodilla y columna vertebral), cardiaca, obstetricia y ginecología, urología, traumatismos y urgencias. No hay que olvidar la posible hipercoagulabilidad residual (embolismos, convulsiones y mortalidad).

\subsubsection{Concentrados de fribrinógeno}

Primera línea de tratamiento en hemorragia con déficit de fibrinógeno documentado (mediante TEG o estudio de coagulación). El límite mínimo hemostático de fibrinógeno es 150-200 $\mathrm{mg} / \mathrm{dl}$ (mayor en embarazo).

\subsubsection{Complejo protombínico (CCP)}

El CCP ha sustituido al plasma para revertir la anticoagulación oral con cumarínicos en paciente grave o cirugía urgente, siempre asociado a vitamina $\mathrm{K}$.

\subsection{Recuperación de sangre autóloga (RSA)}

Sistema de recogida de sangre del campo quirúrgico (intraoperatorio) o drenajes (postoperatorio), para reinfusión posterior. Siempre se debe asegurar la esterilidad, reinfundir en las primeras 6 horas tras la recogida $y$ utilizar filtros de $40 \mathrm{~nm}$.

$\mathrm{Su}$ indicación principal son procedimientos con necesidad transfusional frecuente o con pérdidas impredecibles o esperadas de más del $20 \%$ de volemia; $\mathrm{Hb}$ baja o riesgo alto de hemorragia. Dado su costeefectividad, se ha utilizado en ausencia de otra alternativa disponible; o para disminuir la tasa transfusional (cirugía oncológica, traumatismos abdominales, cesárea o rotura de embarazo ectópico).

\subsection{Manejo de la hemostasia a la cabecera del paciente (TEG/ROTEM)}

Es la mejor guía de evaluación y sustitución dirigida de hemoderivados en coagulopatía sangrante de traumatizados $(36 \%)$ y hemorragia quirúrgica grave (cardiaca, vascular, hepática, obstétrica), donde disminuye la tasa de transfusión y embolias.

\section{Mejorar tolerancia a la anemia}

Evaluación de la capacidad de compensación, prevenir los signos de 
hipoxia y minimizar la necesidad de transfusión.

\section{Discusión}

Nuestro papel como anestesiólogos es fundamental en las estrategias de ahorro de sangre, pues más de la mitad de las transfusiones hospitalarias se realizan en el perioperatorio. El objetivo principal de la optimización es mantener la perfusión tisular, y por lo tanto asegurar la función orgánica. La optimización se debe realizar en un periodo temprano, antes de que se haya producido el daño orgánico.

Recientemente se está implantando un nuevo concepto multimodal, multidisciplinar e individualizado para el manejo integral del paciente quirúrgico. Dentro de este nuevo concepto, el manejo del paciente sangrante (Patient Blood Management) constituye un punto importante, cuyo objetivo principal es reducir o eliminar la necesidad transfusional y mejorar los resultados clínicos.

Este manejo multimodal se basa en los 3 pilares resumidos anteriormente: estímulo de la eritropoyesis o tratamiento de la anemia perioperatoria; reducción del riesgo hemorrágico $\mathrm{y}$ tratamiento activo dirigido del sangrado; y la tolerancia a la anemia perioperatoria, basado en aceptar la anemia normovolémica, criterios «restrictivos» de transfusión, uso de sangre autóloga y mejora de la reserva cardiopulmonar. Estos tres pilares se deben aplicar en tres momentos: preoperatorio, intraoperatorio $\mathrm{y}$ postoperatorio.

Todas las sociedades científicas implicadas destacan la importancia del tratamiento etiológico de la anemia preoperatoria siempre que sea posible y recuerdan que la transfusión presenta sus complicaciones, riesgos y costes adicionales. Actualmente, todos los profesionales implicados debemos colaborar en la optimización y manejo individualizado de nuestros pacientes con el objetivo de conseguir los mejores resultados clínicos, con mayor calidad, eficiencia y satisfacción de pacientes y profesionales.

\section{Bibliografía}

1. Brammar A, Nicholson A, Trivella M, Smith AF. Perioperative fluid volume optimization following proximal femoral fracture. Cocharane review. 2013. (ubmed) (HTML) (ㄹDF)

2. Eelex A, Baikady RR. Perioperative Blood Management. Indian J Anaesth 2017; 61 (6): 456-462 (PubMed) (HTML1) (HTML2)

3. García Erce JA, Laso Morales MJ. «Patient blood management» en la Vía Clínica de Recuperación Intensificada en Cirugía

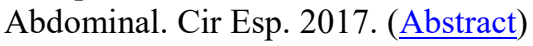

4. Hidalgo, Gomez Luque A., Ferrandis R., Llau JV., De Andrés J., Gomar C., Sierra P., Castillo J., Torres LM. Rev. Española Anestesiología

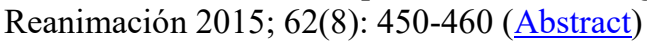

5. Leal-Noval SR, Muñoz $M$, Asuero $M$, Contreras E, García-Erce JA, Llau JV et al. The 2013 Seville Consensus Document on alternatives to allogenic blood transfusion. An update on the Seville Document. Rev Esp Anestesiol Reanim. 2013; 60(5): 243-4. (PubMed)

6. Lobo SL, Mendes CL, Rezende E, Dias FS. Optimizing perioperative hemodynamics: what is new? Curr Opin Crit Care 2013; 19: 346-352. (PubMed)

7. Llau JV, Acosta FJ, Escolar G, FernándezMondéjar E, Guasch E et al. Multidisciplinary consensus document on the management of massive haemorrhage (HEMOMAS document). Med Intensiva 2015; 39(8): 483-504. (TML)

8. Ministerio de Sanidad, Política Social e Igualdad. Grupo de trabajo sobre Recuperación intensificada en Cirugía Abdominal. Vía clínica de Programa de Recuperación intensificada en cirugía abdominal (RICA). 2015. ( $\underline{\mathrm{PDF}})$

9. Muñoz JL, Gabaldón T, Miranda E, Berrio DL, Ruiz Tovar J, Ronda JM et al. Goaldirected fluid therapy on laparoscopic sleeve 
gastrrectomy in morbidly obese patiens. Obese surgery. Obes Surg. 2016; 26(11):2648-2653. ( $\underline{\text { PubMed }}$ ( $\underline{\text { HTML }}$

10. Navarro LHC, Bloomstone JA, AnderJOC, Canneson M, Rocc GD,Gant TJ, et al. Perioperative fluid therapy: A statement from the international Fluid optimization group. Perioper. Medm Lond. Engl. 2015; 4:3. ( $\underline{\text { PubMed }}$ ( $\underline{\text { HTML1) }}$ ( $\underline{\text { HTML2) }}$ ( $\underline{\text { PDF }})$

11. Ochagavía A, Baigorri F, Mesquida J, Ayuela JM, Ferrándiz A, García $X$ et al. Hemodynamic monitoring in the critically patient. Med Intensiva. 2014; 38(3): 154- 169. ( $\underline{\text { PubMed) }}$ (

12. Pearse RM, Moreno RP, Bauer P, Pelosi P, Metnitz P, Spies C et al. Mortality after surgery in Europe: a 7 day cohort study. Lancet 2012; 380: 1059-65. ( PubMed) (HTML) (HTML2) (PDF)

13. Ripollés J, Espinosa A, Martínez-Hurtado E, Abad-Gurumeta A, Casans-Francés R,
Fernández Pérez $\mathrm{C}$ et al. Intraoperative goal directed hemodynamic therapy in noncardiac surgery: a systematic rewiew and metaanaylisis. Rev Bras Anestesiol 2016; 66 (5): 513-28. ( $\underline{\text { PubMed }}$ )

14. http://anesthesiology.pubs.asahq.org/article. aspx? articleid $=2088825 \# 70955587$

15. http://www.jcvaonline.com/article/S1053$\underline{0770(17) 30552-9 / \mathrm{pdf}}$

Correspondencia al autor

María Pérez Herrero

mapeherrero@gmail.com

FEA Anestesiología y Reanimación.

Hospital Clínico Universitario de Valladolid.

Aceptado para blog en febrero de 2018. 